\title{
Echo in a Single Molecule
}

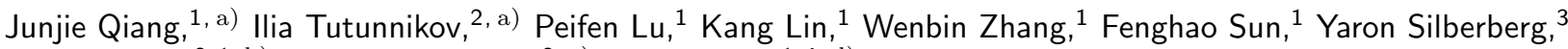 \\ Yehiam Prior, 2,1, b) Ilya Sh. Averbukh, $2, \mathrm{c})$ and Jian $\mathrm{Wu}^{1,4,}$ d) \\ 1) State Key Laboratory of Precision Spectroscopy, East China Normal University, Shanghai 200062, \\ China \\ ${ }^{2)}$ AMOS and Department of Chemical and Biological Physics, Weizmann Institute of Science, Rehovot 7610001, \\ Israel \\ 3) AMOS and Department of Physics of Complex Systems, Weizmann Institute of Science, Rehovot 7610001, \\ Israel \\ ${ }^{4)}$ Collaborative Innovation Center of Extreme Optics, Shanxi University, Taiyuan, Shanxi 030006, \\ China
}

Echo is a ubiquitous phenomenon found in many physical systems, ranging from spins in magnetic fields to particle beams in hadron accelerators. It is typically observed in inhomogeneously broadened ensembles of nonlinear objects, and is used to eliminate the effects of environmental-induced dephasing, enabling observation of proper, inherent object properties. Here, we report experimental observation of quantum wave packet echoes in a single isolated molecule. In contrast to conventional echoes, here the entire dephasing-rephasing cycle occurs within a single molecule without any inhomogeneous spread of molecular properties, or any interaction with the environment. In our experiments, we use a short laser pulse to impulsively excite a vibrational wave packet in an anharmonic molecular potential, and observe its oscillations and eventual dispersion with time. A second delayed pulsed excitation is applied, giving rise to an echo - a partial recovery of the initial coherent wavepacket. The vibrational dynamics of single molecules is visualized by time-delayed probe pulse dissociating them one at a time. Two mechanisms for the echo formation are discussed - ac Stark-induced molecular potential shaking and creation of depletion-induced "hole" in the nuclear spatial distribution. Interplay between the optically induced echoes and quantum revivals of the vibrational wave packets is observed and theoretically analyzed. The single molecule wave packet echoes may lead to the development of new tools for probing ultrafast intramolecular processes in various molecules.

Echoes in physical systems, from their inception by Erwin Hahn in 1950 (1), 2), were born within the framework of spins embedded in an inhomogeneous environment, and are typically used to measure dephasing rates due to the local interaction of the spins (molecules) with their surroundings. Following the original discovery of echoes in ensembles of spins excited by pulsed magnetic fields, many different types of echoes have been observed, including photon echoes (3, 4) and their mechanical analogs (5), cyclotron echoes (6), plasma-wave echoes (7), cold atoms echoes in optical traps (8-10), echoes in cavity QED (11), echoes in particle accelerators (12 15), and more recently - echoes in a gas of rotating molecules $(16-21)$. In general, an ensemble of nonlinear objects (spins, molecules, plasma, beam of particles, etc.) is impulsively excited by an external stimulus resulting in a prompt coherent response. Since each member of the ensemble evolves at a different frequency, the molecular response disappears as the members of the ensemble step out of phase. However, and this is the essence of the echo phenomenon, the effect of the first excitation is not lost, and it is possible to retrieve it. By applying a second pulsed excitation and waiting a time period equal to the delay between the two excitations, a response signal emerges again, and is referred to as an "echo". In general (but not in our case, see below), interaction with the environment causes loss of coherence, and reduces the echo signal amplitude. This reduction in the echo amplitude provides information on the environmentally-induced decoherence. The echo in an ensemble of nonlinear objects should be distinguished from quantum revivals. In contrast to echoes, the quan-

\footnotetext{
a) JQ and IT contributed equally to this work.

b) Electronic mail: yehiam.prior@weizmann.ac.il

c) Electronic mail: ilya.averbukh@weizmann.ac.il

d) Electronic mail: jwu@phy.ecnu.edu.cn
}

tum revivals appear after a single-pulse excitation, and their period depends only on the intrinsic properties of the molecular energy spectrum (22) 25).

Distinctly and in parallel, the interest in single molecule spectroscopy has increased starting with the seminal works of W. E. Moerner (26) and Michel Orrit (27), and eventually leading to super-resolution and super-sensitive optical microscopy. Single molecules embedded in thin films are selected spectrally by narrow band CW lasers within homogeneously broadened lines, or isolated spatially by observing dilute samples where only one molecule lies within the field of view of the microscope. An alternative approach to observing single molecules is to measure them embedded within individual quantum dots, and then spectral or spatial resolutions are not required, enabling time domain experiments (28, 29). Ultrafast experiments based on sensitive fluorescence detection allowed observation of various coherent excitations of vibrational wave packets in single molecules (30) (for a review see (31) and a more recent work (32) and references therein). Coherent oscillatory signals from molecular vibrational wave packets impulsively excited by short pulses are typically washed out on the femtosecond time scale after several oscillations. Such a decay is not caused by the ensemble inhomogeneity, and it has nothing to do with interaction with the environment on such a short time scale. The collapse of the coherent transients originates from intramolecular dephasing of multiple vibrational states forming the wave packet in a single molecule. This is, actually, a quantum phenomenon, as oscillations of a single nonlinear classical oscillator never collapse, and last "forever".

The other type of "single particle experiment" is typically an interference experiment where a single electron, or a single photon, passes through a double slit and interference is observed due to the interaction of this single particle with the double slit $((33,34)$, and references therein). Each particle passes the double slit and reaches 
the screen behind it without any other particles present, and the probability of its hitting the screen at a specific location is provided by quantum mechanics. The probability distribution can be measured by repeating the measurement many times, every time with a new single particle. These experiments are known as "single photon" or "single electron" interference experiments, and this is exactly the type of experiment we are presenting here, but in our case the interference happens in the time domain. The molecules interact with the laser fields one at a time and are individually measured. As in similar experiments, in order to see the distributions, the measurement is repeated many times.

In the present paper, we show that it is possible to partially overcome the effects of intramolecular dephasing and recur the signal by inducing an echo in a single isolated molecule. We report experimental observation of the quantum Wave Packet Echo (WPE) at the level of a single molecule, and provide a detailed theoretical analysis of this phenomenon. In our experiments, using an ultrashort femtosecond laser pulse, we create a localized vibrational wave packet in an argon dimer cation. This wave packet oscillates back and forth in the ion potential well and disperses completely due to the spread of the oscillation frequencies within the wave packet. Next, after a time delay of $t_{k}$, a second laser pulse is applied. We demonstrate that an impulsive molecular response (echo) indeed emerges at time $2 t_{k}$ as a result of the rephasing of the components of the wave packet. The entire sequence of excitation, dispersion, re-excitation and the WPE occurs within a single isolated molecule. The ability to observe a single molecule event stems from our detection methodology of COLTRIMS (COLd Target Recoil Ion Momentum Spectroscopy) (35, 36) (see below and in the Methods section), where the dissociation fragments of each single molecule are individually detected following its dissociation. The observed WPE is a generic phenomenon that can, in principle, be observed by imaging nuclear dynamics in a variety of molecules (37, 38), and in other impulsively excited isolated quantum systems. Our choice of weakly bound argon dimers is motivated by their relatively low vibrational frequencies which simplifies the time-resolved measurements.

Classical counterpart of the quantum WPE. While the vibrational WPE in a single molecule is a quantum phenomenon, a classical phase space analysis of the evolution of an ensemble of molecules provides useful insights into the mechanism of the echo formation. With this qualifying remark in mind, we now consider the vibrational dynamics in an ensemble of non-interacting homonuclear diatomic molecules. The vibrational motion of each molecule is modeled as motion of a classical point particle having mass $M / 2$ in a one-dimensional anharmonic potential, where $M$ is the mass of each atom. Motivated by our experiments, we consider the $\mathrm{I}(1 / 2 u)$ potential of argon dimer cation $\mathrm{Ar}_{2}^{+}$(Fig. 22a). The potential curve is approximated by a Morse potential, $V(R)=D\left(1-\exp \left[-a\left(R-R_{0}^{+}\right)\right]\right)^{2}$, where $R$ is the interatomic separation, and the parameters are $D=0.048$, $a=0.85$, and $R_{0}^{+}=4.56$ (all in atomic units) (39). The Hamiltonian governing the motion of each molecule is $\mathcal{H}(p, R)=p^{2} / M+V(R)$, where $p$ is the momentum associated with the vibrational motion. To imitate the initial state created by an instantaneous ionization of $\mathrm{Ar}_{2}$, the initial distribution is described by a non-equilibrium phase space distribution (Fig. 11, light red)

$$
\rho \propto \exp \left[-\left(R-R_{0}\right)^{2} / 2 \sigma^{2}\right] \exp \left[-\sigma^{2} p^{2} / 2\right],
$$

where $R_{0}$ is the equilibrium internuclear separation of $\mathrm{Ar}_{2}$, and $\sigma^{2}$ is the ground state variance of $\mathrm{Ar}_{2}$ (obtained by Gaussian function fit).
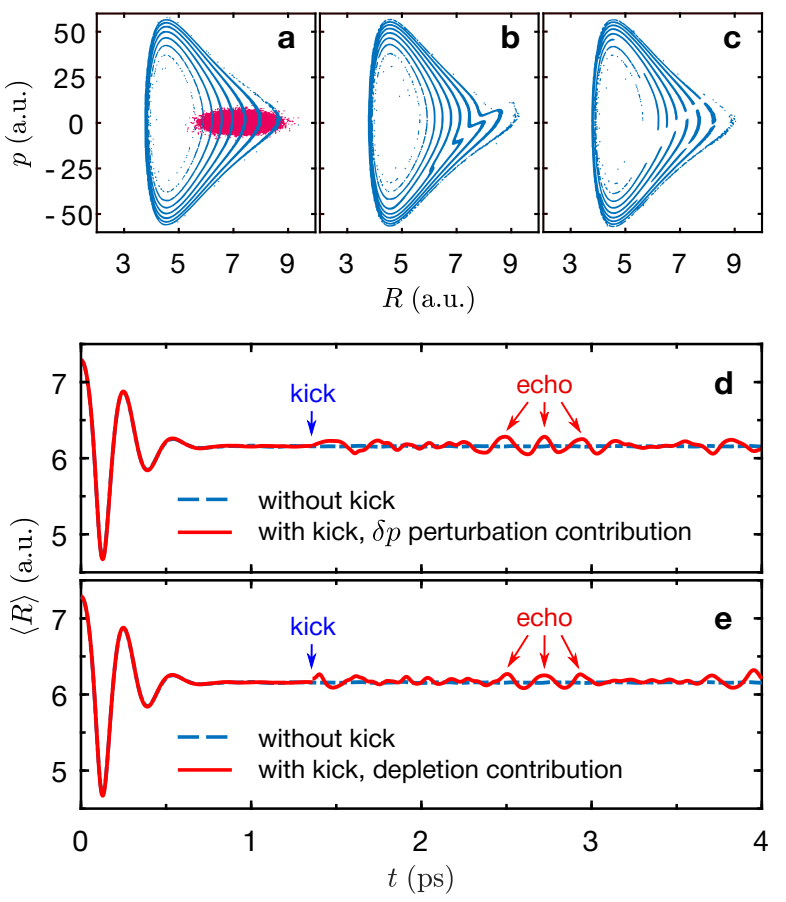

Fig. 1. Phase space dynamics. The temporal evolution of the non-equilibrium phase space distribution (Eq. 1) of an ensemble of molecules $\left(N=10^{5}\right)$. a, Initial distribution (in light red) and filamented phase space distribution just before the second kick at $t_{k}=1.36 \mathrm{ps}$ (in blue), $\mathbf{b}$, The distribution shortly after a kick consisting of a uniform momentum increment in each molecule by $\delta p=1$ a.u., and $\mathbf{c}$, The distribution shortly after a kick consisting of depleting molecules within the strip of 5.5 a.u. $<\mathrm{R}<6.1$ a.u.. The next two panels illustrate the ensemble averaged interatomic separation, $\langle R\rangle(t)$ and the echo response at $t \approx 2 t_{k}$ without (dashed blue) and with (solid red) a kick: d, depicts the contribution of the $\delta p$ perturbation (corresponding to the filamented phase space in panel b) and e, depicts the contribution of the particles depletion (corresponding to the filamented phase space in panel c).

In an anharmonic potential, the frequency (period) of oscillations is energy-dependent. As a result, the initial smooth phase-space distribution (Fig. 1a, light red) evolves with time into a spiral-like structure (blue) and gradually fills the accessible phase space as shown in Fig. 1a. This filamentation of the phase space results in an increasing number of spiral turns which become thinner to conserve the volume, as is known in stellar systems $(\sqrt[40]{ })$ and in accelerator physics $(41)$. To induce the echo response, a second excitation ("kick") is applied at $t=t_{k}$ when the filamentation is well-developed. In our experiments, the kick is provided by an ultrashort laser pulse that disturbs the molecules in two ways: (i) it instantaneously changes the nuclei momentum due to the coordinate-dependent ac Stark shift of the molecular potential, and (ii) it transfers a fraction of the ground state molecules to the excited electronic state via the optical absorption process. In the latter case, the kick drills a 
"hole" in the spatial distribution (a vertical strip in the phase space distribution), centered at the resonance position (42). We consider the two mechanisms separately and show that both lead to a subsequent echo response at $t=2 t_{k}$ as shown in Figs. 1 $(\mathrm{d}, \mathrm{e})$.

In the case of the first mechanism, a kick caused by coordinate-dependent ac Stark shift of the molecular electronic terms is modeled by an instantaneous uniform addition of momentum $\delta p$ to all molecules. The kick is followed by formation of sharp "tips" on each branch of the spiral (Fig. 1b). Due to the spread in their relative velocities in the anharmonic potential the tips desynchronize with time. Here, we use the ensemble-averaged time-dependent internuclear separation, $\langle R\rangle(t)$ as an indicator for the distribution spreading. The initial filamentation of the phase space leads to apparent decaying oscillations of $\langle R\rangle(t)$ as the distribution spreads. The kick at $t=t_{k}$ gives rise to instantaneous response in $\langle R\rangle(t)$ which then decays as well, but at time $t \approx 2 t_{k}$ the tips meet again resulting in a transient spatial inhomogeneity and an echo signal can be clearly observed (see Fig. 11). The described mechanism is similar to the one responsible for formation of transverse beam echoes predicted and observed in particle accelerators, see (1215).

In the case of the second mechanism, we assume that the kick depletes the population in some spatial interval, but does not affect the vibrational motion. In other words, the pulse carves out a strip from the phase space distribution by instantaneously removing all the particles within this strip. This process mimics optical absorption and creates a "hole" on each branch of the spiral. Figure 1. shows the phase space distribution shortly after the instantaneous depletion within the strip 5.5 a.u. $<R<$ 6.1 a.u.. These holes move relative to each other and desynchronize with time (similar to the tips discussed above). As in the previous case, the holes meet again at $t=2 t_{k}$, leading to the echo response. Figure 1p shows the echo signal induced by the second mechanism. As noted, while the single molecule wave packet cannot be described classically, the classical phase-space analysis sheds light on the origin of the echo and suggests the two mechanisms leading to its formation. Results of a full quantum mechanical simulation are presented below.

Experimental methodology. The experiments were performed on $\mathrm{Ar}_{2}$ in an ultra-high vacuum chamber of COLTRIMS (35, 36). In this methodology, the kinetic energy release (KER) of the dissociated molecules is extracted by analyzing the ions collected after ionization by a strong ultrashort laser pulse (Fig. 22). The $\mathrm{Ar}_{2}$ dimers are generated in a supersonic molecular beam by expanding room temperature argon gas through a $30 \mu \mathrm{m}$ nozzle under a backing pressure of 1.6 bar. A fraction of about $1 \%$ dimers was produced with respect to the atomic monomers. The estimated vibrational temperature is $T_{\mathrm{vib}}=10 \mathrm{~K}$, so it is safe to assume that the entire population is in the ground state (see Methods section). A femtosecond laser pulse, derived from a Ti:sapphire multi-pass amplifier $(790 \mathrm{~nm}, 25 \mathrm{fs}, 10 \mathrm{kHz})$, is split and frequency doubled using a $\beta$-BBO crystal to produce $395 \mathrm{~nm}$ pump and kick pulses, and a third pulse at the fundamental wavelength of $790 \mathrm{~nm}$ to probe the vibrational dynamics of the molecules. The peak intensities used in these experiments were $2.0 \times 10^{14} \mathrm{~W} / \mathrm{cm}^{2}$, $1.1 \times 10^{13} \mathrm{~W} / \mathrm{cm}^{2}$ and $8.0 \times 10^{13} \mathrm{~W} / \mathrm{cm}^{2}$ for the pump, kick, and probe pulses, respectively. The durations of the pump, kick, and probe pulses (FWHM) were measured at the focus to be $75 \mathrm{fs}, 110 \mathrm{fs}$, and $70 \mathrm{fs}$, respectively. The three pulses were collinearly recombined and focused by a concave silver mirror $(f=75 \mathrm{~mm})$ onto the gas beam inside the vacuum chamber. The time delays between the three pulses were controlled by two motorized delay stages. The 3D momenta and kinetic energy of the $\mathrm{Ar}^{+}$ produced in dissociation of the $\mathrm{Ar}_{2}^{+}$were reconstructed from the time-of-flights (TOF) and positions of the impacts on the Multi-Channel Plate (MCP) detector.

Based on the driving pressure, temperature of the gas source and the detailed geometry of the jet system, the density of the dimers in the laser beam focal volume is estimated to be $2 \times 10^{6}$ molecules $/ \mathrm{cm}^{3}$. For the laser beam diameter at the focus of $\approx 7 \mu \mathrm{m}$ with a Rayleigh length of $\approx 80 \mu \mathrm{m}$, there is less than one molecule on average in our laser interaction volume at any given time. With the low rate of events, there is much less than a single event per laser shot. At the given laser rep-rate of $10 \mathrm{kHz}$, the molecules in the interaction volume are refreshed after each laser shot. The COLTRIMS way of measurement, in particular the low event rate and individual particle detection, guarantees that only one molecule is measured in any given event. Furthermore, if by chance, more than one molecule had been dissociated, due to the low detection efficiency of the MCP, typically only one $\mathrm{Ar}^{+}$fragment will be detected, leading, again, to a single molecule event. Note that if (with very low probability in our experiments) two ionic fragments were detected, the event is later rejected in the off-line data analysis. The $\mathrm{Ar}^{+}$ions coming from the dissociation of the $\mathrm{Ar}_{2}^{+}$have a kinetic energy of a few eV and are clearly distinguishable from those coming from ionization of the Ar monomers. The monomer events are excluded by examining the kinetic energy of the detected $\mathrm{Ar}^{+}$.

The interaction of the laser pulses with $\mathrm{Ar}_{2}$ is depicted in Fig. 23. The ground electronic state of $\mathrm{Ar}_{2}^{+}$is binding with a dissociation energy of $1.34 \mathrm{eV}$ and equilibrium interatomic separation $R_{0}^{+}=4.5$ a.u. (43), which is smaller than the equilibrium separation of $\mathrm{Ar}_{2}\left(R_{0}=7.2\right.$ a.u.) (44). The pump pulse at $395 \mathrm{~nm}$ creates a vibrational wave packet in the $\mathrm{I}(1 / 2 u)$ state of $\mathrm{Ar}_{2}^{+}$centered at the equilibrium distance $R_{0}$ (point $\mathrm{A}$ ) of the neutral $\mathrm{Ar}_{2}$ dimer. To follow the vibrational dynamics, the wave packet is then dissociated by a time delayed probe pulse with a delay covering the range of $-0.25 \mathrm{ps}$ $<t_{p}<4$ ps. The probe pulse $(790 \mathrm{~nm})$ "catches" the oscillating molecule at point B, as illustrated in Fig. 2a, leading to dissociation of $\mathrm{Ar}_{2}^{+}$via $\mathrm{Ar}_{2}^{+} \rightarrow \mathrm{Ar}^{+}+\mathrm{Ar}$, denoted as $\mathrm{Ar}_{2}(1,0)$ channel. The produced $\mathrm{Ar}^{+}$fragment has KER in the range of $0.7 \mathrm{eV} \leq \mathrm{KER} \leq 1.6 \mathrm{eV}$.

In order to obtain a good signal-to-noise ratio in the measurement of KER vs $t_{p}$, the measurements are repeated many times for different time delays. Thus, the raw data consists of many uncorrelated single events, and for each event KER and $t_{p}$ are recorded. In our experiments, typically the time delay is scanned with 10 fs resolution. At each time delay many laser shots are counted, and the entire sequence is repeated till the desired signalto-noise ratio is obtained. Figure $3 c$ is a result of 30 cycles scanning the whole range of $t_{p}$, where each cycle consisted of $\approx 8$ million laser shots resulting in $\approx 50,000$ usable events where one molecule was dissociated and 


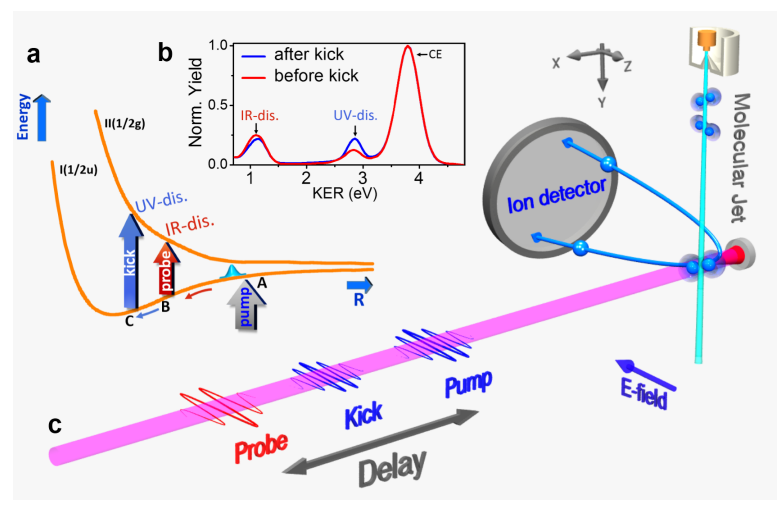

Fig. 2. Experimental setup. a, A coherent nuclear wave packet is launched using the pump pulse which excites a neutral argon dimer $\mathrm{Ar}_{2}$ (not shown) to the $\mathrm{I}(1 / 2 u)$ potential of the ion $\mathrm{Ar}_{2}^{+}$at point $\mathrm{A}$. The wave packet oscillates in the bound I $(1 / 2 u)$ potential, and is later dissociated by the excitation of the ion to its dissociating II $(1 / 2 g)$ potential curve. The excitation to the II $(1 / 2 g)$ state can be done by either the probe pulse at $790 \mathrm{~nm}$ at point $\mathrm{B}$, or by the kick pulse at $395 \mathrm{~nm}$ at point $\mathrm{C}$, leading to dissociation via the $\mathrm{Ar}_{2}(1,0)$ channel with different KER ranging from $0.7 \mathrm{eV}$ to $3.2 \mathrm{eV}$ as shown in b, In the above, "IR-dis." and "UV-dis." refer to dissociation induced by $790 \mathrm{~nm}$ and $395 \mathrm{~nm}$ pulses, respectively. At higher energies, close to $4 \mathrm{eV}$, the Coulombexplosion (CE) double ionization peak is seen. c, Schematic illustration of the experimental apparatus.

recorded at a time.

Figures 3(a-c) depicts the gradual buildup of the interference pattern in the KER distribution as more and more events are accumulated. Animated movies of this buildup may be seen in the Supplementary Movie. The developing interference structure is a time-domain analog of the buildup of spatial interference fringes while scattering single electrons through a double slit (see (33) and movies therein).

As the pump pulse ionizes an argon dimer, it creates a vibrational wave packet that oscillates under the $\mathrm{I}(1 / 2 u)$ potential. This wave packet is a superposition of multiple eigenstates of the $\mathrm{I}(1 / 2 u)$ potential as determined by the original equilibrium state of the argon neutral dimer and the laser parameters. The spatial localization of the wave packet persists for several oscillations. However, due to the anharmonicity of the potential, eventually dephasing takes over, leading to the collapse of the wave packet. Figure $3 \mathrm{~d}$ shows the yield of $\operatorname{Ar}_{2}(1,0)$ channel as a function of $t_{p}$. The curve is obtained by integrating the KER distribution in the range $0.7 \mathrm{eV} \leq \mathrm{KER} \leq 1.6 \mathrm{eV}$. The blue curve in Fig. $4 \mathrm{~b}$ reflects the vibrational motion of the wave packet. The three pronounced oscillations with a period of $\approx 245 \pm 10$ fs near $t_{p}=0$ correspond to the initial oscillatory motion of the still localized wave packet on the $\mathrm{I}(1 / 2 u)$ potential (Fig. 2a). With the vibrational revival time of $\mathrm{Ar}_{2}^{+}$being $14 \mathrm{ps}$, the oscillations at doubled frequency near probe delay of $t_{p}=3.5 \mathrm{ps}$ correspond to quarter-revival (24), (45). Higher fractional revivals are observed, as well, including the $1 / 8$ th revival near $t_{p}=1.8 \mathrm{ps}$ and the $1 / 6$ th revival near $t_{p}=2.3 \mathrm{ps}$. The oscillation period of the $1 / 8$ th revival is $63 \pm 10 \mathrm{fs}$ and that of $1 / 6$ th revival is $82 \pm 10 \mathrm{fs}$.

Observation of echoes in a single molecule. The echo is induced by a kick pulse arriving at $t_{k}=1.36$ ps after the pump. The kick pulse $(395 \mathrm{~nm})$ also cou-

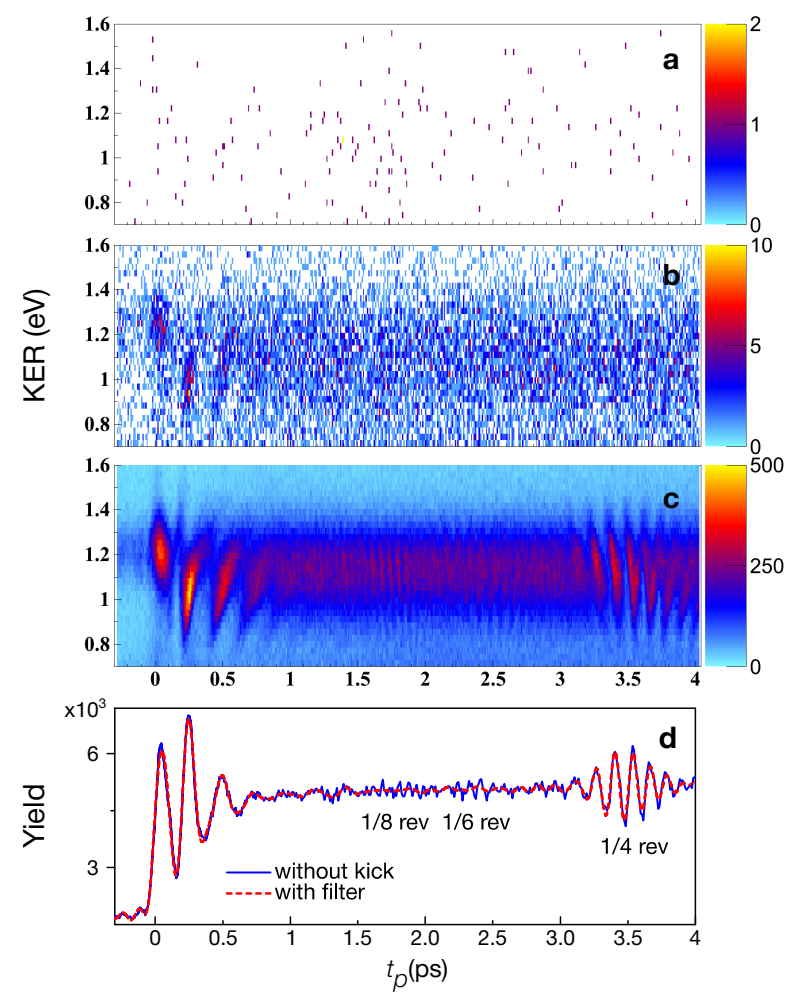

Fig. 3. KER distribution buildup. Kinetic energy distribution of molecular fragments $\left(\operatorname{Ar}_{2}(1,0)\right.$ channel) as a function of the probe delay $t_{p}$ following excitation by a single femtosecond pulse at $790 \mathrm{~nm}$. In panels $(\mathbf{a}, \mathbf{b})$ the events are added: a, 150 events, $\mathbf{b}, 1.5 \times 10^{4}$ events. Panel $\mathbf{c}$ depicts the full measurement after adding the single molecule events from all 30 cycles $\left(1.5 \times 10^{6}\right.$ events $)$. The instantaneous response to the pulse excitation is clearly visible, as is the quarter revival around 3.5 ps. d, Yield of $\operatorname{Ar}_{2}(1,0)$ channel at energies $(0.7 \mathrm{eV} \leq \mathrm{KER} \leq 1.6 \mathrm{eV})$ induced by the probe pulse. Dashed red curve is the same data after applying the low-pass filter (see text).

ples the $\mathrm{I}(1 / 2 u)$ and $\mathrm{II}(1 / 2 g)$ states (at point C, Fig. 2a), leading to partial dissociation of the same parent ion with fragments having KER in the range of $2.5 \mathrm{eV} \leq \mathrm{KER} \leq 3.2 \mathrm{eV}$, higher than the energy band at $0.7 \mathrm{eV} \leq \mathrm{KER} \leq 1.6 \mathrm{eV}$ induced by the probe and discussed above (see Fig. 4h). The disjoint KER energy ranges enable unambiguous assignment of each dissociation event to either the probe or the kick pulse. Figure $4 \mathrm{~b}$ shows the kick-induced (solid black) and probe-induced (solid blue) yields of $\operatorname{Ar}_{2}(1,0)$ channel as a function of probe delay, $t_{p}$. The kick perturbs the collapsed wave packet and also partially transfers the population from the $\mathrm{I}(1 / 2 u)$ state at point $\mathrm{C}$ to the $\mathrm{II}(1 / 2 g)$ state, thus triggering the two mechanisms for echo formation discussed above.

In addition to the prominent fractional revivals, the blue curve in Figure $4 \mathrm{~b}$ shows three new peaks corresponding to the echo signal (showed in the inset and indicated by arrows at $2.16 \mathrm{ps}, 2.41 \mathrm{ps}$ and $2.66 \mathrm{ps}$ ). The period of these new oscillations is $\approx 245 \pm 10 \mathrm{fs}$, which is approximately the period of the original oscillations. Thus, the kick has induced a delayed partial rephasing of the original vibrational wave packet. For delays $0<t_{p}<t_{k}$ the probe precedes the kick, therefore the corresponding kick induced (black curve) and probe induced (blue curve) induced yields of the $\mathrm{Ar}_{2}(1,0)$ channel are out of phase. The drop in the probe induced yield (blue curve) 


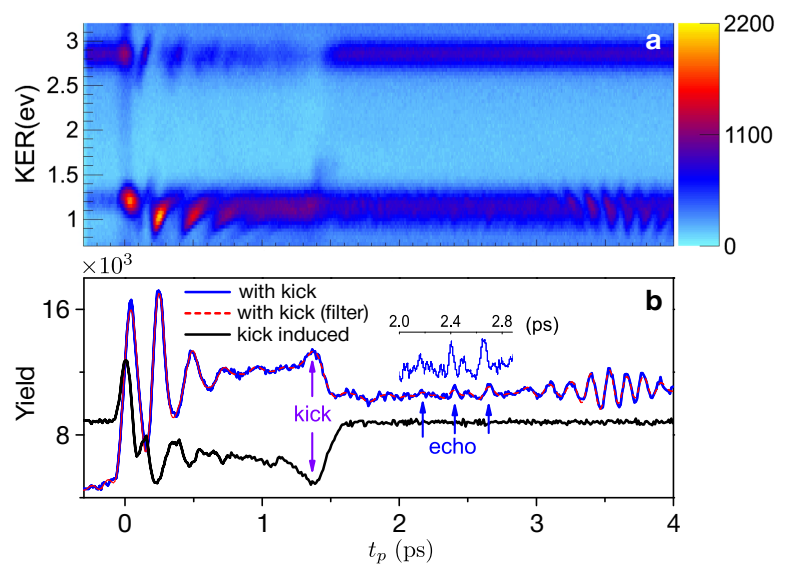

Fig. 4. KER distribution and yield of $\operatorname{Ar}_{2}(1,0)$ channel as a function of the probe delay in a presence of "kick" pulse. Panels $\mathbf{a}$ and $\mathbf{b}$ are similar to Figs. 3(c,d), but in the presence of kick pulse. The kick at $395 \mathrm{~nm}$ produces a higher energy $(2.5 \mathrm{eV} \leq \mathrm{KER} \leq 3.2 \mathrm{eV})$ yield in the $\mathrm{Ar}_{2}(1,0)$ channel. Solid black curve is the kick-induced yield.

around $t_{p}=t_{k}$ results from the partial depletion of the wave packet by the kick pulse. For longer probe delays $t_{p}>t_{k}$ the kick (at a fixed delay of $t_{k}=1.36 \mathrm{ps}$ ) precedes the probe and thus the kick-induced yield (black) remains constant.

Although the echo signal partially temporally overlaps the $1 / 6$ th fractional revival (around probe delay $t_{p}=2.3$ ps), it can be distinguished from the fractional revival because of their different oscillation freqiencies. While the oscillation period of the echo signal is the same as that of the original excitation, the frequency of the $1 / 6$ th revival oscillations is three times that of the fundamental frequency. By applying a low-pass filter, it is possible to significantly suppress the contribution of the $1 / 6$ th revival. Details of the filtering procedure are presented in the Methods Section. As is shown in Fig. $3 \mathrm{~d}$ (dashed red curve), the low-pass filter removes the high-frequency components of signal, while retaining the contributions of the fundamental and second-harmonic frequencies, which highlights the visibility of the echo signal as displayed in Fig. 4 p (dashed red curve). The observation of these echoes is the main experimental result of the current work.

Quantum mechanical analysis of the WPE. We carried out quantum mechanical simulations of the observed WPE using two versions of the two-state molecular model. In the first (simplified) one, we allowed dipole coupling between the $\mathrm{I}(1 / 2 u)$ and the II $(1 / 2 g)$ states of $\mathrm{Ar}_{2}^{+}$only for the kick pulse, and calculated the expectation value of the interatomic separation as a function of time, $\langle R\rangle(t)$. These results are used for comparing the quantum WPE in a single molecule, and classical echo in an ensemble of many molecules. In the second, more sophisticated model, both the kick and the probe pulses are allowed to couple the two molecular states. This model fits well the experimental configuration as described in Section III. Here, the probe pulse produces an outgoing wave packet representing the products of dissociation which is then analyzed for constructing the KER spectra as a function of the probe delay. In both versions of the quantum model, we assume that the initial state is prepared by instantaneous ionization of $\mathrm{Ar}_{2}$

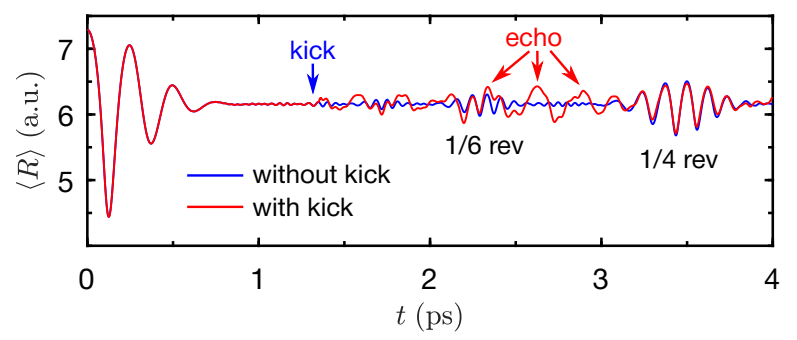

Fig. 5. Quantum mechanical simulations of the echo dynamics. Expectation value of the interatomic separation, $\langle R\rangle(t) \equiv\left\langle\psi_{1}|R| \psi_{1}\right\rangle /\left\langle\psi_{1} \mid \psi_{1}\right\rangle$ as a function of time with (blue) and without (red) the kick. This result is calculated without the dissociating probe. The kick transfers $\approx 9 \%$ of the population from the ground to the excited state. The parameters of the kick pulse are: $I_{0 k}=10^{12} \mathrm{~W} / \mathrm{cm}^{2}, \omega_{k}=2 \times 0.0569$ a.u. $(400 \mathrm{~nm}), t_{k}=1.36 \mathrm{ps}$ and $\mathrm{FWHM}_{k}=10 \mathrm{fs}$.

by the pump pulse, i.e. the ground state of the neutral dimer is projected onto the $\mathrm{I}(1 / 2 u)$ potential without any change. The time evolution of the two-state system is described by a pair of coupled Schrödinger equations (46, 47)

$$
\left\{\begin{array}{l}
i \dot{\psi}_{1}=\left[T+V_{1}(R)\right] \psi_{1}-E(t) \mu_{\|}(R) \psi_{2} \\
i \dot{\psi}_{2}=\left[T+V_{2}(R)\right] \psi_{2}-E(t) \mu_{\|}(R) \psi_{1}
\end{array}\right.
$$

where $T$ is the kinetic energy operator and indices 1,2 refer to the $\mathrm{I}(1 / 2 u)$ and II $(1 / 2 g)$ potentials $\left(V_{1,2}(R)\right)$, respectively. The off-diagonal terms describe the dipole coupling between the two states and are equal to the product of the coupling field $E(t)$ and the coordinate dependent transition dipole moment $\mu_{\|}(R)$. In the numerical calculation we add absorbing potentials to the diagonal terms (not shown here, see Methods Section) to avoid spurious reflections from the edge of the spatial grid. The electric fields of the kick and probe pulses are given by

$$
E_{j}(t)=E_{0 j} \cos \left(\omega_{j} t\right) \exp \left[-2 \ln 2\left(\frac{t-t_{j}}{\mathrm{FWHM}_{j}}\right)^{2}\right],
$$

where $E_{0 k}, E_{0 p}$ are the amplitudes of the fields, $\omega_{k}, \omega_{p}$ are the carrier frequencies, $t_{k}, t_{p}$ are the delays of the kick and probe pulses, respectively, and $\mathrm{FWHM}_{k, p}$ refers to the temporal intensity profiles of the kick and probe. The coordinate-dependent transition dipole moment of $\mathrm{Ar}_{2}^{+}$ is $\mu_{\|}(R)=R / 2(48)$ (see Methods Section for details). The coupling field for the first version of the quantum model is $E(t)=E_{k}$, while for the second one it is $E(t)=$ $E_{k}+E_{p}$.

In the quantum simulations, we model the kick by adding an off-diagonal term to the Hamiltonian describing the time evolution of the two-state system. Such coupling acts as a source for the two echo-inducing mechanisms. Unlike the classical case, here it is not possible to separate the contributions of the momentum shift and depletion. However, as is shown in (42), under the conditions of our experiment the two state model does include both contributions - the creation of the "dynamical hole" (depletion mechanism) and the momentum shift (ac Stark shift mechanism). Details of the models and calculations are provided in the Methods Section.

Figures 5 and 6 show the results of the two simulations. Figures $6 \mathrm{a}$ and $6 \mathrm{p}$ display the KER distributions as a 

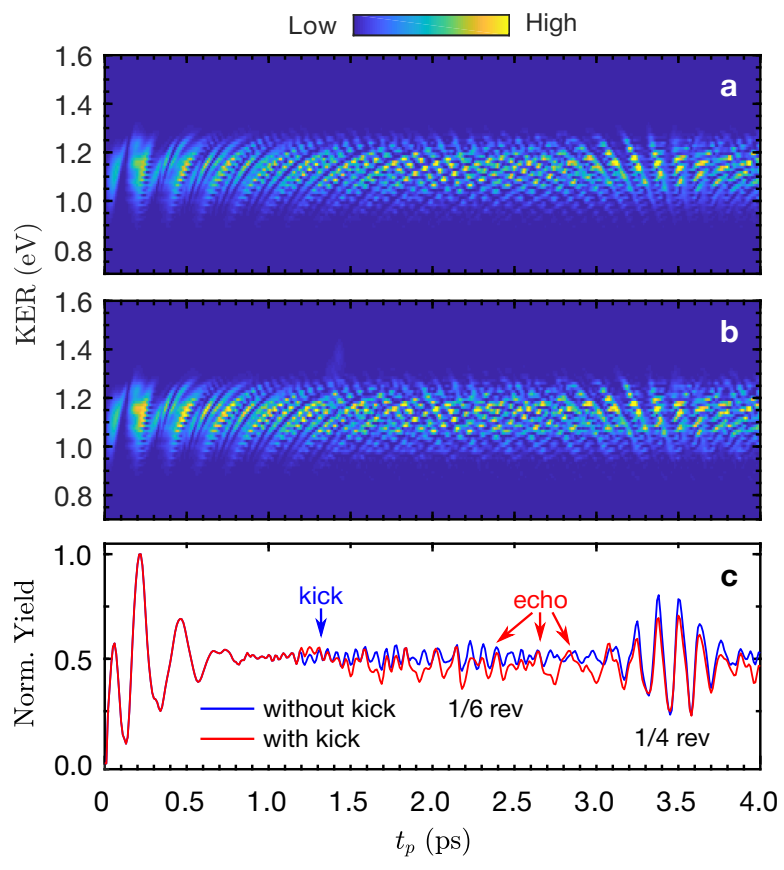

Fig. 6. KER distributions as a function of probe delay. Kinetic energy distribution of molecular fragments $\left(\operatorname{Ar}_{2}(1,0)\right.$ channel) $\mathbf{a}$, without and $\mathbf{b}$, in the presence of the kick pulse. The kick arrives at a delay $t_{k}=1.36 \mathrm{ps}$. c, The normalized probe-induced yield of the $\operatorname{Ar}_{2}(1,0)$ channel as a function of the probe arrival time, $t_{p}$, with (red curve) and without (blue curve) the kick pulse. The parameters of the probe pulse: $I_{0 p}=10^{12} \mathrm{~W} / \mathrm{cm}^{2}, \omega_{p}=0.0569$ a.u. $(800 \mathrm{~nm})$ and $\mathrm{FWHM}_{p}=70 \mathrm{fs}$. The kick pulse parameters are the same as in Fig. 5

function of the probe delay, $t_{p}$ without (Fig. 6a) and with the kick pulse (Fig. 6p). The normalized yield (Fig. 6c) is obtained by integrating the KER distributions in the range of $0.7 \mathrm{eV}<\mathrm{KER}<1.6 \mathrm{eV}$. In analogy to the classical analysis (Figs. 11(d,e)), both Figs. 5 and 6 . show the change in the periodicity of the signal around $t \approx 2 t_{k}$, corresponding to the echo response.

In our simulations, the intensities of the pulses are smaller than the experimentally measured ones. It is mainly because in the experiments most of the molecules do not experience the maximal nominal intensity of the pulse because of their location within the spatially Gaussian intensity distribution. Also, we found that the 10 fs duration (broader bandwidth) of the kick pulse results (for the adopted pulse intensities) in a better visibility of the simulated echo signals (e.g. in Figs. $6(b, c))$, probably due to the fact that the pulses used in the experiment also carry a broad bandwidth, but are longer due to chirping. Further work will be required to address these issues quantitatively.

Discussion. Here we introduce WPE in a single isolated molecule and illustrate it in a vibrating argon dimer cation. Unlike the conventional echoes, which are the collective response of inhomogeneous ensembles of many molecules each under different environmental conditions, here the entire echo cycle occurs within a single isolated molecule. A coherent vibrational wave packet is prepared by instantaneous ionization of a neutral argon dimer, which then oscillates in the anharmonic ground state potential of the cation. The spreading of the generated wave packet and the disappearance of the initial coherent oscillations are due to the dispersion of the vibrational frequencies within the excited wave packet. After a time delay, a second impulsive excitation is applied, inducing a subsequent echo response showing a (partial) recovery of the initial coherent oscillations. This intramolecular dynamics was measured by breaking the molecular ion in a COLTRIMS setup where the kinetic energy of probe-induced dissociation products was measured as a function of the probe delay. The essence of our detection method is that only events where a single molecule (ion) has been exploded are counted. The experimental results are compared with fully quantum mechanical simulations, and analogy is drawn with a related classical echo phenomenon. The classical phase space analysis reveals a connection between the echo in highly excited vibrating molecules and transverse echoes in accelerator beams of particles (12 15). The analysis suggests two mechanisms for the vibrational quantum WPE: one is based on impulsive shaking of the molecular potential due to non-resonant Stark effect, while the second one relies on impulsive local depletion of the vibrational wave packet.

This classical analysis provides intuitive hints about the origin of the vibrational echo, however a proper description of the WPE requires a full quantum-mechanical treatment. It is interesting to compare the present experiment with our previous studies on rotational alignment echoes (18), which used a similar experimental approach. In the latter case, the rotational temperature of the molecules was high enough such that the dynamics could be treated classically, and therefore the observed rotational alignment echo could be properly described by the classical phase-space analysis. In the present case, all the argon dimers are initially in the ground vibrational state, and are then prepared in the same pure vibrational wave packet on the ion potential. The only reason for the dephasing of this wave packet, and its partial rephasing after a delayed kick is quantum intra-molecular dynamics.

Recent years have shown increased interest in the coherent dynamics of single quantum objects, especially in relation to the problem of storing and retrieving quantum information in quantum network setups. Studies on single electron spin echoes in molecular systems (49) and isolated quantum dots (50, 51), as well as photon echoes from a few molecules (52), were reported. In these experiments, the environmental inhomogeneity is handled by averaging the observations over many seconds during which the environment changes many times, typically on a microsecond time scale. As is clearly and explicitly pointed out by the authors $(49 \sqrt[52]{52})$, these "single molecule" time-averaged observations are completely equivalent to standard echoes observed from an inhomogeneous ensemble of many molecules, where the ensemble averages are replaced by time averages over a single object. In contrast, in our experiments the entire echo cycle of excitation and observation is completed in an isolated single molecule on ultrafast time scale.

The present demonstration is done on simple dimer molecules, and the method of detection is photoninduced dissociation followed by analysis of the fragments impinging on an MCP detector. Naturally, such a detection method is limited to a small number of particles hitting the detector, and hence to small molecules. However, the phenomenon of WPEs in a single molecule is generic, and with advanced detection methods, one 
may envisage probing the internal dynamics of larger molecules, shedding light on ultrafast intramolecular processes.

\section{METHODS}

Molecular beam. Experimentally, a dilute molecular beam was obtained by supersonicly expanding argon gas through a $30 \mu \mathrm{m}$ nozzle into the ultrahigh vacuum chamber of the COLTRIMS apparatus. As is common in similar molecular beam experiments, the density of molecule in the focal volume is estimated by considering the driving pressure and temperature of the gas source, the pumping capability, and the geometry of the system. The vibrational temperature of the molecules shares a similar value with translation temperature, which can be estimated using $T_{\text {vib }} \approx T_{\text {trans }}=\Delta p^{2} /\left[4 \ln (4) k_{\mathrm{B}} m\right]$, where $k_{\mathrm{B}}$ is the Boltzmann constant, and $\Delta p$ and $\mathrm{m}$ are the FWHM of the momentum distribution in the jet direction and mass of the singly ionized $\mathrm{Ar}_{2}^{+}$, respectively. In our experiment, a momentum width in the jet direction was measured to be $\Delta p \approx 5.1$ a.u. of $\operatorname{Ar}_{2}^{+}$cations created by pump pulse polarized orthogonally to the gas jet. Thus, the vibrational temperature in the interaction region of the argon dimers is estimated to be $T_{\mathrm{vib}}=10 \mathrm{~K}$. At this temperature it is safe to assume that the entire population is in the ground state.

Signal filtering. We applied a low-pass filter to curves in Figs. 3d and 4b. Using a discrete Fourier transform, we obtained the frequency spectrum of the signal, containing the fundamental vibration frequency, as well as quarter and $1 / 6$ th revivals frequencies, denoted $f, 2 f$ and $3 f$, respectively. Then we retain only the frequencies below $2 f$ in order to remove the $1 / 6$ th revival and higher frequency components. Finally, we reconstructed the signal in the time domain by applying the inverse discrete Fourier transform. Figure 7 shows the magnified portion of Figs. 3d and $4 \mathrm{~b}$.

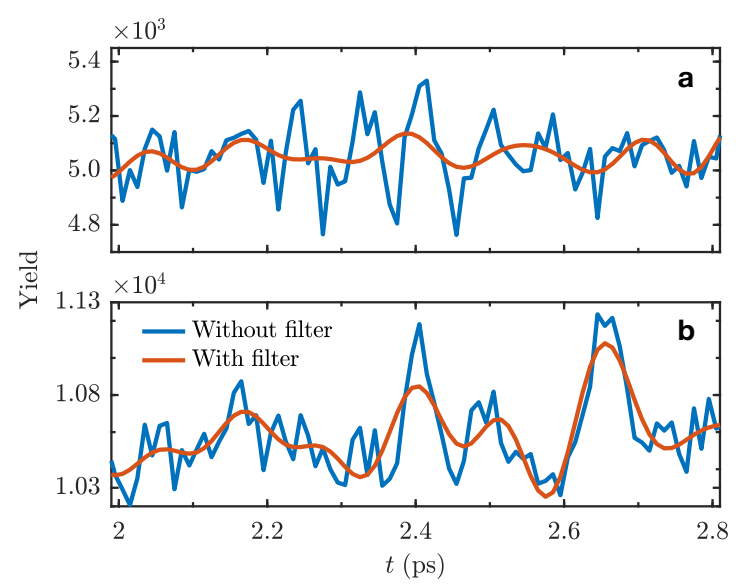

Fig. 7. Magnified portion of Figs. 3g and 4b. a, Yield without the kick pulse. b, Yield with the kick pulse. Both curves represent the yield of $0.7 \mathrm{eV} \leq \mathrm{KER} \leq 1.6 \mathrm{eV}$ channel.

Numerical scheme. For solving the system of coupled Schrödinger equations (Eq. 2), we discretize the spatial variable, $R$ such that $\psi_{1}$ and $\psi_{2}$ are represented as column vectors in $\mathbf{C}^{N}$ and $V_{1}, V_{2}$ are diagonal $N \times N$ matrices, where $N$ is the size of the spatial grid. The discrete fourth order accurate approximation of the Laplacian operator is given by

$$
\frac{\partial^{2} \psi}{\partial R^{2}} \approx \frac{-\psi(R-2 \Delta R)+16 \psi(R-\Delta R)-30 \psi(R)+16 \psi(R+\Delta R)-\psi(R+2 \Delta R)}{12(\Delta R)^{2}}
$$

and it is represented by a symmetric, sparse $N \times N$ matrix. Here, $\Delta R$ is the spatial grid step size and we used $\Delta R=0.15$ a.u. The above form assumes that the wavefunction vanishes at the boundaries, $\psi\left(R \leq R_{\min }, t\right)=\psi\left(R \geq R_{\max }, t\right)=0$. To assure this, we chose the grid size and the total propagation time $\left(t=t_{p}+0.3 \mathrm{ps}\right)$, such that the detached probeinduced wave packet does not reach the boundary at all. We also included the absorbing potential near the right edge of the spatial grid

$$
V_{\mathrm{abs}}=-i a \Theta\left(R-R_{\mathrm{b}}\right)\left(\frac{R-R_{\mathrm{b}}}{R_{\max }-R_{\mathrm{b}}}\right)^{2}
$$

Here, $\Theta(\cdot)$ is a unit step function and we used the following parameters: $a=0.01$ a.u., the right grid edge is $R_{\max }=34$ a.u., beginning of the absorbing layer lies at $R_{\mathrm{b}}=32$ a.u. The absorbing boundary is important when the probe pulse arrives after the kick pulse and the kick-induced outgoing wave packet may have enough time to reach the boundary. The off-diagonal elements of the system (Eq. 2) are $E(t) \mu_{\|}(R)$. Strictly speaking, the parallel transition dipole moment, $\mu_{\|}(R)$ equals to $R / 2$ only for the potentials without the spin-orbit coupling (48). Nevertheless, we set $\mu_{\|}(R)=R / 2$, because near the resonance point B (Fig. 2 a) $\mu_{\|} \approx R / 2$. After discretizing the right hand side of Eq. 2, ordinary differential equation solver (based on the fourth order Runge-Kutta algorithm) was used for time propagation. 
KER calculation. To obtain the distribution of the kinetic energy release (KER) as a function of the probe delay, $\xi\left(\mathrm{KER}, t_{p}\right)$ we allowed propagation of the wave packet on both $\mathrm{I}(1 / 2 u)$ and $\mathrm{II}(1 / 2 g)$ potential curves up to $t_{\text {max }}=$ $t_{p}+0.3 \mathrm{ps}$ for each probe pulse delay, $t_{p}$ and computed the momentum space representation of the wavefunctions at this moment. The integration was done in the region $R \in\left[10, R_{\max }\right]$, where the wave packets representing the dissociation products are well separated from the bound part. Then, the probability density for kinetic energy was obtained by

$$
\xi\left(\mathrm{KER}, t_{p}\right) \propto \frac{\sqrt{M}}{p}\left(\left|\tilde{\psi}_{\mathrm{I}(1 / 2 u)}\left(p, t_{\max }\right)\right|^{2}+\left|\tilde{\psi}_{\mathrm{II}(1 / 2 g)}\left(p, t_{\max }\right)\right|^{2}\right)
$$

where the - denotes Fourier transform.

\section{REFERENCES}

${ }^{1}$ E. L. Hahn. Spin echoes. Phys. Rev., 80:580-594, 1950.

${ }^{2}$ E. L. Hahn. Free nuclear induction. Phys. Today, 6(11):4, 1953.

${ }^{3}$ N. A. Kurnit, I. D. Abella, and S. R. Hartmann. Observation of a photon echo. Phys. Rev. Lett., 13:567-568, 1964.

${ }^{4}$ S. Mukamel. Principles of nonlinear optical spectroscopy. Oxford University Press, New York, 1995.

${ }^{5}$ V. P. Chebotayev and B. Ya. Dubetsky. A classical model of the photon echo. Appl. Phys. B, 31(1):45-52, 1983.

${ }^{6}$ R. M. Hill and D. E. Kaplan. Cyclotron resonance echo. Phys. Rev. Lett., 14:1062-1063, 1965.

${ }^{7}$ R. W. Gould, T. M. O'Neil, and J. H. Malmberg. Plasma wave echo. Phys. Rev. Lett., 19:219-222, 1967.

${ }^{8}$ A. Bulatov, A. Kuklov, B. E. Vugmeister, and H. Rabitz. Echo in optical lattices: Stimulated revival of breathing oscillations. Phys. Rev. A, 57:3788-3792, 1998.

${ }^{9}$ F. B. J. Buchkremer, R. Dumke, H. Levsen, G. Birkl, and W. Ertmer. Wave packet echoes in the motion of trapped atoms. Phys. Rev. Lett., 85:3121-3124, 2000.

${ }^{10}$ M. Herrera, T. M. Antonsen, E. Ott, and S. Fishman. Echoes and revival echoes in systems of anharmonically confined atoms. Phys. Rev. A, 86:023613, 2012.

${ }^{11}$ T. Meunier, S. Gleyzes, P. Maioli, A. Auffeves, G. Nogues, M. Brune, J. M. Raimond, and S. Haroche. Rabi oscillations revival induced by time reversal: A test of mesoscopic quantum coherence. Phys. Rev. Lett., 94:010401, 2005.

${ }^{12}$ G. Stupakov. Echo Effect in Hadron Colliders. SSC Report SSCL-579, 1992.

${ }^{13}$ L. K. Spentzouris, J.-F. Ostiguy, and P. L. Colestock. Direct measurement of diffusion rates in high energy synchrotrons using longitudinal beam echoes. Phys. Rev. Lett., 76:620-623, 1996.

${ }^{14} \mathrm{G}$. V. Stupakov. Handbook of Accelerator Physics and Engineering, chapter 2.3.13 - Echo Effects, pages 121-123. World Scientific, Singapore, $2^{\text {nd }}$ edition, 2013.

${ }^{15}$ T. Sen and Y. S. Li. Nonlinear theory of transverse beam echoes. Phys. Rev. Accel. Beams, 21:021002, 2018.

${ }^{16}$ G. Karras, E. Hertz, F. Billard, B. Lavorel, J.-M. Hartmann, O. Faucher, E. Gershnabel, Y. Prior, and I. Sh. Averbukh. Orientation and alignment echoes. Phys. Rev. Lett., 114:153601, 2015.

${ }^{17}$ G. Karras, E. Hertz, F. Billard, B. Lavorel, G. Siour, J.-M. Hartmann, O. Faucher, E. Gershnabel, Y. Prior, and I. Sh. Averbukh. Experimental observation of fractional echoes. Phys. Rev. A, 94:033404, 2016.

${ }^{18}$ K. Lin, P. Lu, J. Ma, X. Gong, Q. Song, Q. Ji, W. Zhang, H. Zeng, J. Wu, G. Karras, G. Siour, J.-M. Hartmann, O. Faucher, E. Gershnabel, Y. Prior, and I. Sh. Averbukh. Echoes in space and time. Phys. Rev. X, 6:041056, 2016.

${ }^{19}$ J. Lu, Y. Zhang, H. Y. Hwang, B. K. Ofori-Okai, S. Fleischer, and K. A. Nelson. Nonlinear two-dimensional terahertz photon echo and rotational spectroscopy in the gas phase. Proc. Natl. Acad. Sci. U.S.A, 113(42):11800-11805, 2016.

${ }^{20}$ D. Rosenberg, R. Damari, S. Kallush, and S. Fleischer. Rotational echoes: Rephasing of centrifugal distortion in laserinduced molecular alignment. J. Phys. Chem. Lett, 8(20):5128$5135,2017$.

${ }^{21}$ D. Rosenberg, R. Damari, and S. Fleischer. Echo spectroscopy in multilevel quantum-mechanical rotors. Phys. Rev. Lett., $121: 234101,2018$.
${ }^{22}$ J. H. Eberly, N. B. Narozhny, and J. J. Sanchez-Mondragon. Periodic spontaneous collapse and revival in a simple quantum model. Phys. Rev. Lett., 44:1323-1326, 1980.

${ }^{23}$ J. Parker and C. R. Stroud. Coherence and decay of Rydberg wave packets. Phys. Rev. Lett., 56:716-719, 1986.

${ }^{24}$ I. Sh. Averbukh and N. F. Perelman. Fractional revivals: Universality in the long-term evolution of quantum wave packets beyond the correspondence principle dynamics. Phys. Lett. A, 139(9):449 - 453, 1989.

${ }^{25} \mathrm{R}$. W. Robinett. Quantum wave packet revivals. Phys. Rep, 392(1):1 - 119, 2004 .

${ }^{26}$ W. E. Moerner and L. Kador. Optical detection and spectroscopy of single molecules in a solid. Phys. Rev. Lett., 62:2535-2538, 1989.

${ }^{27}$ M. Orrit and J. Bernard. Single pentacene molecules detected by fluorescence excitation in a p-terphenyl crystal. Phys. Rev. Lett., 65:2716-2719, 1990.

${ }^{28}$ T. Guenther, C. Lienau, T. Elsaesser, M. Glanemann, V. M. Axt, T. Kuhn, S. Eshlaghi, and A. D. Wieck. Coherent nonlinear optical response of single quantum dots studied by ultrafast nearfield spectroscopy. Phys. Rev. Lett., 89:057401, 2002.

${ }^{29}$ T. Unold, K. Mueller, C. Lienau, T. Elsaesser, and A. D. Wieck. Optical Stark effect in a quantum dot: Ultrafast control of single exciton polarizations. Phys. Rev. Lett., 92:157401, 2004.

${ }^{30}$ D. Brinks, F. D. Stefani, F. Kulzer, R. Hildner, T. H. Taminiau, Y. Avlasevich, K. Müllen, and N. F. van Hulst. Visualizing and controlling vibrational wave packets of single molecules. Nature, 465:905-908, 2010.

${ }^{31}$ D. Brinks, R. Hildner, E. M. H. P. van Dijk, F. D. Stefani, J. B. Nieder, J. Hernando, and N. F. Van Hulst. Ultrafast dynamics of single molecules. Chem. Soc. Rev., 43:2476-2491, 2014.

${ }^{32}$ M. Liebel, C. Toninelli, and N. Van Hulst. Room-temperature ultrafast nonlinear spectroscopy of a single molecule. Nature Photonics, 12:45-49, 2018.

${ }^{33}$ R. Bach, D. Pope, Sy-H. Liou, and H. Batelaan. Controlled double-slit electron diffraction. New Journal of Physics, 15, 2013.

${ }^{34}$ A. Aspect and P. Grangier. The First Single Photon Sources and Single Photon Interference Experiments, pages 3-23. Springer International Publishing, Cham, 2019.

${ }^{35}$ R. Dörner, V. Mergel, O. Jagutzki, L. Spielberger, J. Ullrich, R. Moshammer, and H. Schmidt-Böcking. Cold target recoil ion momentum spectroscopy: a "momentum microscope" to view atomic collision dynamics. Physics Reports, 330(2):95 - 192, 2000 .

${ }^{36}$ J. Ullrich, R. Moshammer, A. Dorn, R. Dörner, L. Ph. H. Schmidt, and H. Schmidt-Böcking. Recoil-ion and electron momentum spectroscopy: reaction-microscopes. Rep. Prog. Phys, 66(9):1463, 2003.

${ }^{37}$ S. De, M. Magrakvelidze, I. A. Bocharova, D. Ray, W. Cao, I. Znakovskaya, H. Li, Z. Wang, G. Laurent, U. Thumm, M. F. Kling, I. V. Litvinyuk, I. Ben-Itzhak, and C. L. Cocke. Following dynamic nuclear wave packets in $\mathrm{N}_{2}, \mathrm{O}_{2}$, and $\mathrm{CO}$ with few-cycle infrared pulses. Phys. Rev. A, 84:043410, 2011.

${ }^{38}$ I. A. Bocharova, A. S. Alnaser, U. Thumm, T. Niederhausen, D. Ray, C. L. Cocke, and I. V. Litvinyuk. Time-resolved Coulomb-explosion imaging of nuclear wave-packet dynamics induced in diatomic molecules by intense few-cycle laser pulses. Phys. Rev. A, 83:013417, Jan 2011.

${ }^{39}$ The three parameters are obtained from fitting the $a b$ initio potential curve (43) with Morse potential.

${ }^{40}$ D. Lynden-Bell. Statistical mechanics of violent relaxation in stellar systems. Mon. Notices Royal Astron. Soc, 136(1):101$121,1967$.

${ }^{41}$ Allan J. Lichtenberg. Phase-space Dynamics of Particles (Wiley 
series in plasma physics). John Wiley \& Sons Inc, 1969.

${ }^{42}$ U. Banin, A. Bartana, S. Ruhman, and R. Kosloff. Impulsive excitation of coherent vibrational motion ground surface dynamics induced by intense short pulses. J. Chem. Phys, 101(10):84618481, 1994.

${ }^{43} \mathrm{~A}$. Wüest and F. Merkt. Potential energy curves of diatomic molecular ions from high-resolution photoelectron spectroscopy. I. The first six electronic states of $\mathrm{Ar}_{2}^{+}$. J. Chem. Phys, 120(2):638-646, 2004.

${ }^{44}$ S. M. Cybulski and R. R. Toczyłowski. Ground state potential energy curves for $\mathrm{He}_{2}, \mathrm{Ne}_{2}, \mathrm{Ar}_{2}, \mathrm{He}-\mathrm{Ne}, \mathrm{He}-\mathrm{Ar}$, and $\mathrm{Ne}-$ Ar: a coupled-cluster study. The Journal of Chemical Physics, 111(23):10520-10528, 1999.

${ }^{45}$ J. Wu, M. Magrakvelidze, A. Vredenborg, L. Ph. H. Schmidt, T. Jahnke, A. Czasch, R. Dörner, and U. Thumm. Steering the nuclear motion in singly ionized argon dimers with mutually detuned laser pulses. Phys. Rev. Lett., 110:033005, Jan 2013.

${ }^{46}$ B. M. Garraway and K. A. Suominen. Wave-packet dynamics: new physics and chemistry in femto-time. Rep. Prog. Phys, 58(4):365, 1995.

${ }^{47} \mathrm{M}$. Magrakvelidze and U. Thumm. Dissociation dynamics of noble-gas dimers in intense two-color IR laser fields. Phys. Rev. A, 88:013413, 2013.

${ }^{48}$ F. X. Gadea and I. Paidarová. Ab initio calculations for $\mathrm{Ar}_{2}^{+}$, $\mathrm{He}_{2}^{+}$and $\mathrm{He}_{3}^{+}$, of interest for the modelling of ionic rare-gas clusters. Chem. Phys., 209(2):281 - 290, 1996.

${ }^{49}$ J. Wrachtrup, C. von Borczyskowski, J. Bernard, R. Brown, and M. Orrit. Hahn echo experiments on a single triplet electron spin. Chemical Physics Letters, 245(2):262 - 267, 1995.

${ }^{50}$ F. H. L. Koppens, K. C. Nowack, and L. M. K. Vandersypen. Spin echo of a single electron spin in a quantum dot. Phys. Rev.
Lett., 100:236802, 2008

${ }^{51}$ D. Press, K. De Greve, P. L. McMahon, T. D. Ladd, B. Friess, C. Schneider, M. Kamp, S. Höfling, A. Forchel, and Y. Yamamoto. Ultrafast optical spin echo in a single quantum dot. Nature Photonics, 4(6):367-370, 2010.

${ }^{52} \mathrm{H}$. Dong and G. R. Fleming. Three-pulse photon echo of finite numbers of molecules: Single-molecule traces. The Journal of Physical Chemistry B, 117(38):11318-11325, 2013.

\section{ACKNOWLEDGMENTS}

We acknowledge useful discussions with Dan Oron, Dekel Raanan and Gennady Stupakov. This work is supported by the National Key R\&D Program of China (Grant No. 2018YFA0306303), the National Natural Science Foundation of China (Grant Nos. 11425416, 11834004, 61690224, 11621404 and 11761141004), the 111 Project of China (Grant No. B12024), the Israel Science Foundation (Grant No. 746/15), the ICORE program "Circle of Light", and the ISF-NSFC (Grant No. 2520/17). I.A. acknowledges support as the Patricia Elman Bildner Professorial Chair, and thanks for the hospitality extended to him by the UBC Department of Physics\&Astronomy where part of this work was carried out. This research was made possible in part by the historic generosity of the Harold Perlman Family. 